\title{
LA ECONOMÍA POPULAR Y SOLIDARIA COMO PROYECTO POLÍTICO POSTNEOLIBERAL DE ECUADOR. CASO: ACUERDO COMERCIAL UNIÓN EUROPEA-ECUADOR
}

POPULAR AND SOLIDARITY ECONOMY AS A POSTNEOLIBERAL POLITICAL PROJECT IN ECUADOR CASE STUDY: EUROPEAN UNION-ECUADOR TRADE AGREEMENT

\section{LOURDES AGUAS}

Recibido 29 de mayo del 2016 Aceptado 2 de septiembre del 2016

* Pontificia Universidad Católica del Ecuador, Quito, Ecuador (maguas302@puce.edu.ec) 



\title{
LA ECONOMÍA POPULAR Y SOLIDARIA COMO PROYECTO POLIITICO POSTNEOLIBERAL DE ECUADOR. CASO: ACUERDO COMERCIAL UNIÓN EUROPEA-ECUADOR
}

\author{
POPULAR AND SOLIDARITY ECONOMY AS A \\ POSTNEOLIBERAL POLITICAL PROJECT IN ECUADOR \\ CASE STUDY: EUROPEAN UNION-ECUADOR TRADE \\ AGREEMENT
}

\section{LOURDES AGUAS}

PALABRAS CLAVE: Economía popular y solidaria, economía social, comercio justo, acuerdo comercial UE-Ecuador, nueva izquierda latinoamericana.

KEY WORDS: Popular and solidarity economy, social economy, fair trade, EUEcuador trade agreement, Latin American new left.

\section{RESUMEN}

La "nueva izquierda" latinoamericana explora discursivamente variaciones a la economía de libre mercado. Dentro de este "proyecto posneoliberal", se hace uso del término "economía popular y solidaria" (EPS) como parte de una estrategia de comercio internacional diferenciadora que abarca al concepto de "comercio justo". Tanto la "EPS" como el "comercio justo" constan en el borra- dor del Acuerdo Comercial Multipartes UE-Ecuador. En este contexto, el artículo presenta tres reflexiones en torno al tema: 1) Los conceptos relacionados con las economías alternativas no son universales. De ahí que se pueda hablar de la existencia de polisemia al contrastar el sentido de la "economía popular y solidaria" en Ecuador con el de la "economía social" en Europa a pesar de que ambos 
incluyen al "comercio justo" como parte constitutiva; 2) Como resultado de esta ambigüedad conceptual, se crean distintas narrativas que estarían presentes en torno al Acuerdo; por tanto, 3) Es necesario delimitar fronteras conceptuales diferenciadoras al momento de estudiar el término "economía de libre mercado"y contrastarlo con el de "economía popular y solidaria" en un momento histórico en el que la supervivencia de "proyectos políticos posneoliberales" está en juego en la región.

\section{ABSTRACT}

The "new left" in Latin America has explored discursively variations of the free market economy. In this "post-neoliberal project", the term popular and solidarity economy (PSE) is used as part of a differentiated foreign Trade strategy that encompasses the concept of "fair trade". Both PSE and fair trade appear in the European Union-Ecuador Trade Agreement draft. In this context, the article presents three considerations: 1) Concepts related to alternative economies are not universal. Thus, there is a condition of polysemy when contrasting the term PSE in Ecuador with the term "social economy" in Europe, despite the fact that both include fair trade as a constitutive element; 2) As a result of this conceptual ambiguity, there are diverse narratives revolving around the Agreement; therefore, 3) It is necessary to delimit the conceptual boundaries between the terms "free-market economy" and "popular and solidarity economy" in a historic moment in which the survival of "post-neoliberal political projects" in the region is at stake.

\section{INTRODUCCIÓN ${ }^{* *}$}

Los movimientos políticos que surgieron en América Latina a finales de 1990 y la primera década del nuevo milenio, como respuesta a las olas de liberalización, la privatización, la crisis económica y el descontento de amplios sectores de la población, son concebidos como

* A lo largo del presente artículo los conceptos clave para el estudio, cuyo significado es relativo, estarán en itálica. la nueva izquierda latinoamericana. Esta nueva izquierda se caracteriza por promover objetivos revolucionarios y de transformación (Calvo, 2009). También es definida como una "izquierda a la defensiva" ávida por explorar variaciones de la economía de mercado y de la democracia 
representativa (Rodríguez, Barret y Chávez, 2008 en Calvo, 2009).

En este contexto, se suscitan reformas constitucionales en varios países latinoamericanos que buscaban conceptualizar e institucionalizar sistemas económicos posneoliberales y visibilizar (Nelms, 2015) nociones como en el caso de Ecuador, la economía popular y solidaria como alternativa a la economía de libre mercado. De ahí que, este concepto se utiliza en el Plan Nacional para el Desarrollo ${ }^{1}$ así como en las políticas públicas que de ahí se derivan. A través de estos documentos se enfatiza, por tanto, la importancia de diferenciar este enfoque alternativo como estrategia de política interna y como parte fundamental de la política exterior ecuatoriana².

Las dificultades surgen al momento de formular estrategias diferenciadas para poner en práctica los mandatos constitucionales, así como, la planificación nacional de desarrollo y las políticas encaminadas a dar una alterna-

\footnotetext{
1 Llamado en Ecuador Plan Nacional del Buen Vivir a partir de 2009.

${ }^{2}$ Cabe mencionar que el documento vigente en cuanto a política exterior ecuatoriana es el Plan Nacional de Política Exterior (PLANEX) 2006-2020 que no contempla el concepto de economía populary solidaria. Sin embargo, se infiere la "estrategia de política exterior ecuatoriana" de otros documentos oficiales provenientes del Ministerio de Relaciones Exteriores y Movilidad Humana, el Ministerio de Comercio Exterior y la Secretaría Técnica de Cooperación Internacional.
}

tiva al enfoque neoliberal. Se toma como ejemplo el Acuerdo de Comercio Multipartes que se negoció entre Ecuador y la Unión Europea a fin de reflexionar sobre sus implicaciones presentes y futuras. Las críticas sobre las cuales se basa este artículo son tres: 1) La polisemia de los conceptos relacionados con las economías alternativas como resultado del contexto en el cual se desarrollan. De aquí se indaga la motivación y el alcance de la noción de economía social en Europa y cómo esta difiere de la de economía populary solidaria en Ecuador; 2) Las distintas narrativas que, como resultado de la ambigüedad conceptual, resultan en el marco del Acuerdo de Comercio Multipartes entre Ecuador y la UE y 3) La necesidad de delimitar fronteras conceptuales diferenciadoras al momento de estudiar la economía de libre mercado y contrastarla con la economía populary solidaria.

Se eligieron documentos a estudiar de manera comparativa en el contexto de la Unión Europea y Ecuador. Estos documentos se relacionan con: la historia de la economía social y la economía popular y solidaria; las declaraciones y normativas referentes al comercio justo en Europa y Ecuador; la legislación, la planificación nacional y los instrumentos de la política exterior ecuatoriana relativos a la economía popular y solidaria y al comercio justo; finalmente, las percepcio- 
nes o posturas de parte de instituciones encargadas en Ecuador y la Unión Europea, así como noticias sobre el Acuerdo negociado provenientes de distintos voceros.

El primer apartado del presente artículo trata las distintas concepciones de las economías alternativas. Se hace una distinción entre la economía social en Europa estudiada a partir de dos actores: 1) El tercer sector u organizaciones de sociedad civil que buscan visibilizar las disparidades presentes en el comercio internacional y que los Estados adquieran compromisos más duraderos con los países en desarrollo y; 2) Los Estados que, si bien no utilizan el concepto de economía social, incorporan recientemente al comercio justo como parte de la política europea de cooperación internacional. En este apartado se hace una reflexión, posteriormente, sobre la economía popular y solidaria como pro- yecto político post neoliberal propuesto por Ecuador.

En el segundo apartado, se analizan las percepciones y narrativas contradictorias del Acuerdo Comercial Multipartes entre Colombia, Perú y Ecuador y la Unión Europea. Se toma como referencia al término tratado de libre comercio y se lo contrasta con el término acuerdo de comercio para el desarrollo.

Finalmente, en el tercer apartado se pretende responder a la pregunta: ¿Cómo dibujar fronteras conceptuales entre la economía de libre mercado y la economía popular y solidaria? Se hace en este sentido, una revisión teórica de los principales aportes en el estudio de las economías alternativas a fin de encontrar elementos diferenciadores que permitan formular estrategias específicas en el ámbito de las políticas públicas, así como del rol del tercer sector tanto en Europa como en Ecuador.

\section{LA POLISEMIA DE LAS ECONOMÍAS ALTERNATIVAS}

A fin de comprender la noción de comercio justo, resulta útil referirse a la obra de Defourny et. al. (2006) para explorar por separado los conceptos de economía social que aplica al caso europeo y economía popular y solidaria relativa al caso latinoamericano. La existencia de dos términos distintos al momento de analizar los orígenes del concepto de comercio justo nos da cuenta de las diferencias geográficas, históricas, lingüísticas y normativas que caracterizan a cada uno de ellos.

Defourny et. al. (2006) señala que todavía no existen suficientes estudios que se enfocan en examinar el concep- 
to de economía social (y sus variantes) y aplique tanto a países desarrollados como a aquellos en desarrollo. En América Latina existen recientemente esfuerzos políticos por visibilizar los otros tipos de economía, por ejemplo, en la Constitución de Ecuador (2008) con el uso del concepto de economía popular y solidaria o en la Constitución de Bolivia (2009) con el concepto de economía comunitaria que procuran, en ambos casos, evidenciar formas no capitalistas de organización económica (Coraggio, 2011), es decir formas de organización que no necesariamente responden únicamente a la motivación del lucro. A pesar de esto, todavía quedan fronteras por definir tanto en el ámbito jurídico como en el de aplicación de políticas públicas, así como en la noción del tercer sector, en lo que respecta la pluralidad del sentido de la economía.
Vale la pena, en este contexto como sugiere Bateson (1972), cambiar la unidad de análisis a fin de ampliar el entendimiento del tema desde una perspectiva sistémica. No convendría limitarse; por tanto, a estudiar el concepto de economía social per se. En cambio, tiene sentido la idea de estudiar la economía social en el ambiente en el cual se desarrolla, puesto que los contextos culturales, sociales, económicos y políticos son diferentes en cada territorio. En esta línea, autores como Fonteneau, Nyssens y Fall (2006) señalan que más allá de la heterogeneidad del llamado sector informal, las actividades económicas que lo caracterizan están incrustadas en determinados contextos, lo cual influencia sus modos de funcionamiento. En definitiva, no existe una única forma de entender las economías alternativas y sería un error universalizarlas como concepto.

\section{LOS DOS ACTORES DE LA ECONOMÍA SOCIAL EN EUROPA (EL TERCER SECTOR Y EL ESTADO)}

\subsection{La economía social y el tercer sector en Europa}

El concepto de economía social, en el contexto europeo, data del siglo XIX y nace de las lenguas latinas europeas. Puede compararse con el sector non-profit (más típico de países de habla inglesa) a pesar que no pueden ser tratados como sinónimos. Dos características fueron identificadas por los estudiosos de la economía social europea a saber: la articulación de una "condición de necesidad"y de una "condición de identidad colectiva" por parte de los grupos de la sociedad civil.

Defourny et. al. (2006) afirma que las olas de desarrollo de la economía so- 
cialen Europa se manifestaron particularmente durante las grandes mutaciones del capitalismo. Constituyeron según los mismos autores la "tercera vía" entre el capitalismo y el centralismo estatal. En su momento, las organizaciones de la economía social jugaron un rol central en la provisión de salud y otros servicios sociales dentro de Europa y permitieron enfrentar los grandes desafíos de la época. Constituyeron en efecto, una suerte de "proto-estado de Bienestar" (a nivel privado) y sentaron las bases de futuras demandas que la sociedad civil planteó posteriormente a nivel estatal.

Surgieron de aquí, alternativas como las "cooperativas de consumo" enfocadas en proveer alimentos básicos a precios más convenientes a familias obreras, gracias a que dichas cooperativas evitaban los intermediarios que imponían condiciones poco favorables de compra y comercialización de sus productos. Dado el contexto de ese entonces de desigualdad de clases en Europa, este ejemplo también podría tomarse a su vez, como la base de lo que más adelante se denominaría comercio justo como una forma de superar las asimetrías, en este caso, entre países denominados desarrollados y países en desarrollo.

Históricamente, la economía social se ocupa de cuestiones éticas en lo que respectan los intercambios de bie- nes y servicios. De ahí surgen por ejemplo, nociones como el precio justo a fin de encontrar soluciones a las preocupaciones sobre equidad y honestidad en el marco de las transacciones comerciales. A escala internacional, poder hacer frente a la explotación laboral dio paso a que entre los años 60 y 70 surgieran las primeras iniciativas de comercio alternativo en Europa.

\subsection{Los Estados europeos y el comercio justo}

Estas cuestiones éticas se evidencian en el comunicado de la Comisión Europea al Consejo relativo al Comercio Justo (1999), donde se indica que el comercio justo forma una parte fundamental de la política europea de cooperación para el desarrollo. Se señala ahí que el comercio justo permite contribuir al desarrollo social duradero de los países del sur.

Se menciona en el mismo documento, que el comercio justo permite acercar a los países desarrollados a los países en desarrollo y facilita una mejor integración de los últimos en la economía mundial en el contexto que los beneficios del comercio y el crecimiento, "no necesariamente repercuten por igual en todos los países y todas las capas de la población de cada país." Por tanto, se buscaría a través de este tipo de comercio reducir las disparidades entre países desarrollados y países en desarro- 
Ilo como resultado del permanente deterioro de los términos de intercambio de los últimos.

Otro elemento importante relacionado con el comercio justo según lo define la UE sería: el precio justo que se traduciría en un incremento de ingresos especialmente para bienes que se consideran "commodities" para los países en desarrollo, en beneficio de pequeños agricultores o productores a pequeña escala que dependen excesivamente de intermediarios. A pesar de no ser una obligación, según la comunicación, las iniciativas de comercio justo pueden incluir opciones de prefinanciamiento y contratos que proporcionen a los productores seguridad en el largo plazo.

Una tercera característica de la definición europea de comercio justo sería la finalidad o motivación de este tipo de comercio ya que se indica que, "las ganancias obtenidas del comercio justo han de repercutir en la comunidad en su totalidad y no en un beneficio personal."

El comercio justo según el modelo europeo nace y se nutre de organizaciones no gubernamentales (tercer sector), por lo que no representa un compromiso público (en otras palabras de "ayuda oficial" o "ayuda al comercio"), sino un compromiso prácticamente privado en su totalidad, (con excepción de las normativas de compras públicas) en donde los consumidores europeos se convier- ten en cooperantes a través de este tipo de iniciativas ${ }^{3}$.

Una cuarta característica del comercio justo en Europa estaría asociada al etiquetado a pesar que se reconoce, que gran parte de los productos comercializados en tiendas de comercio justo no se etiquetan, en cuyo caso la compra se basa en la confianza. Este tipo de relaciones responden a lo que se concibe como comercio justo tradicional, producto de obras de asistencia que nacieron en la década del 60 y 70 en Europa.

A pesar que el comercio justo como concepto se menciona en varios espacios en la UE, no existe legislación específica para el comercio justo que abarque los 28 Estados miembros. Por tanto, la aplicación del comercio justo continúa siendo una competencia nacional o regional (Fair Trade Advocacy Office, 2016).

El reconocimiento más significativo del comercio justo, hasta la actualidad, provino del Parlamento Europeo en su Resolución sobre "Comercio Justo y Desarrollo" votada en 2006. El mayor logro de esta resolución fue solicitar a la Comisión Europea que examine la manera en la cual, el comercio justo se convierta en

${ }^{3}$ Así lo indica la Comunicación: "Los productos del comercio justo llegan a los consumidores por varias vías. Todas ellas pasan por iniciativas privadas, son las más habituales el movimiento tradicional de comercio justo (que incluye las organizaciones comerciales alternativas)". 


\section{LA ECONOMÍA POPULAR Y SOLIDARIA COMO PROYECTO POLÍTICO \\ POSTNEOLIBERAL DE ECUADOR. CASO: ACUERDO COMERCIAL UNIÓN \\ EUROPEA-ECUADOR}

un modelo para una política comercial sostenible capaz de estimular un comercio equilibrado norte-sur. Sin embargo, la posterior respuesta de la Comisión no cubrió todas las demandas.

Además, en 2009 la Comisión Europea publicó un comunicado titulado "Comercio justo y sistemas no gubernamentales de garantía de la sostenibilidad comercial". Dicho comunicado abarcaba una definición más detallada del concepto de comercio justo ${ }^{4}$, no obstante dio cuenta más bien de un enfoque pasivo por parte de los Estados europeos sobre el tema, pues se plantea explícitamente la importancia de "mantener el carácter no gubernamental de las organizaciones que ponen en práctica estos sistemas". En otras palabras, se

${ }^{4}$ El concepto de comercio justo incluye los siguientes criterios: "1) la fijación de un precio justo que cubra los costes de producción y de subsistencia de manera sostenible, así como la posibilidad de abonar cantidades a cuenta a los productores; 2) el establecimiento de relaciones a largo plazo entre productores y distribuidores; 3) la transparencia y trazabilidad a lo largo de toda la cadena de suministro; 4) el respeto de los convenios fundamentales de la Organización Internacional del Trabajo (OIT), sobre todo los relativos al trabajo digno, la libertad sindical y las normas de trabajo; 5) el respeto de los derechos humanos, el ambiente y los métodos tradicionales; 6) el refuerzo de las capacidades de producción y el desarrollo del acceso de los productores al mercado; 7) la sensibilización de los agentes y consumidores sobre el funcionamiento y los objetivos del comercio justo; 8) el establecimiento de un proceso de certificación; 9) la evaluación del impacto de las actividades comerciales." deja en manos del tercer sector la aplicación y fomento del comercio justo. La Comisión señala que el Movimiento de Comercio Justo 5 podrá responder mejor a los asuntos relativos a este tema que los mismos Estados. Al hacer esto, se concibe al comercio justo en términos de garantías de la sostenibilidad comercial en lugar de una forma alternativa de hacer comercio (Fair Trade Advocacy Office, 2016).

Al momento de revisar la política comercial de la UE (Comisión Europea, 2014) se observa que se prioriza la liberalización económica, con el supuesto que una política activa de libre comercio puede mejorar las perspectivas de crecimiento y abrir nuevas oportunidades comerciales en terceros países. Se asocia la liberalización del comercio con la innovación y el fomento de la productividad. Sin embargo, no se hace ninguna mención explícita al concepto de comercio justo por separado.

El único momento en el que los Estados europeos juegan un rol directo respecto al comercio justo es en el ámbito de las compras públicas. En la normativa europea que rige las compras públicas (Parlamento Europeo, 2012) se resalta la importancia de la coherencia

${ }^{5}$ El movimiento de comercio justo en Europa abarca las siguientes instituciones: Fairtrade International, the World Fair Trade Organization and the World Fair Trade Organization-Europe. 
de las autoridades públicas en lo que respecta a la cooperación internacional y el comercio internacional. Asimismo, de manera más general, en el documento informativo de la UE titulado "Comprender las políticas de la Unión Europea: Cooperación Internacional y Desarrollo" publicado en 2014, se resalta la necesidad de reforzar la coherencia entre las relaciones exteriores y la cooperación al desarrollo. De aquí se puede inferir que no basta con que los países donantes incrementen los montos destinados a Ayuda Oficial para el DesarroIlo si no se complementa esto también con otro tipo de acciones relativas al comercio. (el documento sugiere que las preferencias arancelarias constituyen una fuente fundamental de apoyo para reducir la pobreza de los países en desarrollo, por ejemplo).

De todo lo anteriormente mencionado se puede concluir que los Estados europeos no tienen actualmente una legislación comprensiva que promueva y regule el comercio justo. Por tanto, el comercio alternativo constituye una iniciativa de tipo voluntario que el tercer sector puede o no acoger como parte de la ayuda no oficial al desarroIlo. El concepto de economía social no se utiliza a nivel de actores estatales puesto que, contradiría la política exterior europea que tiene un claro enfoque neoliberal.

\section{LA ECONOMÍA POPULAR Y SOLIDARIA COMO PROYECTO POLÍTICO. CONTRADICCIONES EN LA POLÍTICA EXTERIOR ECUATORIANA Y LA POLIITICA INTERNA}

El proyecto político en Ecuador plasmado en la Constitución de 2008, pretendía como señala Nelms (2015) conceptualizar e institucionalizar un sistema económico posneoliberal, que se relaciona explícitamente a valores y principios sociales alternativos como: la solidaridad, la cooperación, la asistencia mutua, la igualdad, entre otros. Sin embargo, señala Nelms, múltiples preocu- paciones surgieron al momento de delimitar el concepto de economía popular y solidaria y poner en práctica sus principios a través de modelos organizacionales, apoyos institucionales y regímenes regulatorios apropiados para estos compromisos. Esto derivó en cuestionamientos sobre la burocracia, la experticia, la transformación estatal y la supervivencia del proyecto político. 


\subsection{La retórica de la economía popular y solidaria y del comercio justo en Ecuador}

En la década de los noventa, algunos autores abandonan la noción de sector informal y la remplazan por economía popular (sobre todo en América Latina y África), ya que afirman que la segunda es más operacional que la primera dado que daría cabida a otras formas de interpretar la realidad socio-económica de los países en desarrollo. (Didier, 2010). La noción de lo solidario emerge de un discurso transnacional en el seno de la comunidad académica, movimientos sociales, agencias de cooperación para el desarrollo, instituciones multilaterales y actores estatales. De ahí que entre 1990 y 2000 aproximadamente, el concepto de economía solidaria se convierta no únicamente en un referente conceptual en la academia, sino también en una categoría utilizada por algunos Estados en el ámbito de las políticas públicas y la gobernabilidad (Nelms, 2015) ${ }^{6}$.

En Ecuador el comercio justo pertenece de manera explícita, a la economía populary solidaria. Este concepto fue visibilizado por primera vez en la Constitución de 2008, específicamente en el Art. 304, referente a la política comercial

${ }^{6}$ Otros pensadores importantes en América Latina incluyen a Luis Razeto, José Luis Coraggio que dicta en el numeral 5: "Impulsar el desarrollo de las economías de escala y del comercio justo." y en el Art. 336 referente a los Intercambios Económicos y el Comercio Justo donde se señala: "El Estado impulsará y velará por el comercio justo como medio de acceso a bienes y servicios de calidad, que minimice las distorsiones de la intermediación y promueva la sustentabilidad".

Según la obra de Dieterich (2007), la economía solidaria formaría parte de un nuevo proyecto histórico y sería una de las características de la civilización poscapitalista. El elemento diferenciador de la economía solidaria con respecto al modelo económico vigente, según Dieterich (2007), radicaría en la redefinición del "valor" de los bienes y servicios (que se determina, por ejemplo, a través de la energía o información) como antítesis de la "economía crematística".

Por tanto, más allá de la concepción europea de la economía social representada sustancialmente por el tercer sector, en el caso de América Latina la economía solidaria y el comercio justo formarían parte de un proyecto político y el Estado se convertiría en su principal vocero y promotor. De ahí que, por ejemplo, en los Planes del Buen Vivir (2009-2013) y (2013-2017), se determinen los objetivos 11 y 8 relacionados con el establecimiento y consolidación de 
"un sistema económico, social, solidario, de forma sostenible".

En 2012 se define a través de Ley Orgánica de Economía Popular y Solidaria de Ecuador (LOEPS), a la economía populary solidaria como:

...la forma de organización económica, donde sus integrantes, individual o colectivamente, organizan y desarrollan procesos de producción, intercambio, comercialización, financiamiento y consumo de bienes y servicios, para satisfacer necesidades y generar ingresos, basadas en relaciones de solidaridad, cooperación y reciprocidad, privilegiando al trabajo y al ser humano como sujeto y fin de su actividad, orientada al buen vivir, en armonía con la naturaleza, por sobre la apropiación, el lucro y la acumulación de capital.

Otra distinción importante, que recalca la Ley en el Art. 5 es el concepto de acto económico solidario como:

Los actos que efectúen con sus miembros las organizaciones a las que se refiere esta Ley, dentro del ejercicio de las actividades propias de su objeto social, no constituyen actos de comercio o civiles sino actos solidarios y se sujetarán a la presente Ley.

Así, dentro de las políticas sociales formuladas por el Estado ecuatoriano y su legislación correspondiente, se resaltan como valores de la "nueva izquier- da": la cooperación, como antítesis de la libre competencia, la solidaridad y la reciprocidad como una forma de incorporar la figura del otro, en las relaciones entre individuos en oposición al individualismo hobbesiano característico de la modernidad europea.

Asimismo, dentro del estudio de la economía alternativa como parte de las estrategias de política exterior ecuatoriana, la Secretaría Técnica de Cooperación Internacional de Ecuador (SETECI, 2015) en su publicación titulada "Diccionario de Cooperación Internacional" define al comercio justo como: "una alternativa distinta al modelo convencional de comercio basado en la construcción de alianzas entre productores y consumidores..." y lo ubica como parte constitutiva de los procesos de integración regional Latinoamericana en donde se buscaría la "construcción de regímenes comerciales justos y de complementariedades en cuanto a las capacidades de cada país". La misma institución identifica dentro de los lineamientos prioritarios para la cooperación internacional en Ecuador y de la política 8.9 del PNBV "Profundizar las relaciones del Estado con el sector popular y solidario", para la cual se plantean lineamientos específicos como: la creación de un sistema de información de los actores de la economía popular, el impulso de la formación técnica para 


\section{LA ECONOMÍA POPULAR Y SOLIDARIA COMO PROYECTO POLÍTICO \\ POSTNEOLIBERAL DE ECUADOR. CASO: ACUERDO COMERCIAL UNIÓN

dichos actores, el fomento de la asociatividad, entre otros ${ }^{7}$.

Asimismo, se pueden rescatar algunos ejemplos que dan cuenta de cómo el comercio justo y la economía alternativa constituyen una parte fundamental de la imagen con la cual Ecuador anhela posicionarse en el mundo ${ }^{8}$. así, en la quinta edición de la revista "Cooperamos" de SETECI (2012) se menciona al comercio justo y la nueva arquitectura financiera donde se recalca "la importancia de generar un nuevo orden económico internacional que debe sustentarse en un nuevo esquema de comercio justo y solidario con las economías en desarrollo y en una nueva arquitectura financiera."

\footnotetext{
${ }^{7}$ A continuación, otros ejemplos. Dentro de la política 7.9 relativa a "Promover patrones de consumo conscientes, sostenibles y eficientes con criterio de suficiencia dentro de los límites del planeta" recalca la necesidad "Establecer y fortalecer espacios de comercialización de bienes y servicios sustentables, en particular de productos orgánicos, con pertinencia cultural y criterios de comercio justo, social y solidario, en las áreas urbanas y rurales". Y finalmente la política 10.5 que dicta la necesidad de "Fortalecer la economía popular y solidaria, y las micro, pequeñas y medianas empresas en la estructura productiva." cuyos principales lineamientos incluyen: el fortalecimiento de las MIPYMES y el impulso del sector financiero populary solidario.

8 Tomando como referencia la intervención de Ecuador en la Cumbre Río + 20, se utiliza al comerciojusto dentro del discurso que los países del norte deben promoverlo y contemplar compensaciones por daños y contingencias (Falconí, 2012) para fomentar la complementariedad entre las naciones.
}

Lo que busca el Estado ecuatoriano en los últimos años, es plantear una política exterior diferenciada. Precisamente esto le permitiría al Ecuador que se lo reconozca como parte de la nueva izquierda latinoamericana. De esta forma, alcanzaría lo que según Browning (2015) se conoce como seguridad ontológica. Es decir, en un mundo globalizado (liberalizado), la búsqueda de identidad nacional ya no se da únicamente a través de la guerra, sino que se da a través de la construcción de narrativas planificadas que permiten que los Estados "vendan" una imagen positiva y se posicionen, de esta forma, en el mundo. El uso discursivo de la economía populary solidaria serviría, en parte a este propósito.

En resumen, en el caso de Ecuador, el proyecto político posneoliberal fue caracterizado con valores como la solidaridad, la reciprocidad y la cooperación como característicos de una economía alternativa y en la política exterior se acompañó de conceptos como autonomía, soberanía y dignidad. Todas estas nociones fueron útiles para construir una narrativa distinta a la que profesa el libre-mercado, la individualidad y la competencia. Permitiría, por tanto, identificarse con los debates que surgen en la nueva izquierda en la región que, como señalan Chávez, Rodríguez y Barret (2008) van más allá de temas específicos como la igualdad económica y la democracia. 


\subsection{Dificultades al momento de establecer estrategias diferenciadoras para la economía popular y solidaria en Ecuador}

En el caso latinoamericano, el concepto de sector informal fue el que concentró la atención de los estudiosos durante muchos años. Se le atribuye el "descubrimiento" de este término a Hart K., antropólogo inglés, quien en 1973 publica en un informe en el marco de la Organización Mundial del Trabajo, sus reflexiones sobre la economía en Kenia y distingue los ingresos provenientes de "trabajo independiente", de aquellos basados en el "salario" (Palmer, 2004). El problema de los países en desarrollo, según el autor, no era tanto el desempleo sino la existencia de un gran grupo de trabajadores pobres cuyas actividades no eran reconocidas ni protegidas o reglamentadas por el Estado.

La Organización Internacional del Trabajo (2016) define actualmente a la economía informal como "todas las actividades económicas realizadas por los trabajadores y unidades económicas que no están cubiertos o que están insuficientemente cubiertos -en la legislación o en la práctica- por acuerdos formales." Para Fonteneau (en Defourny J. et. al. (2006), algunas características del sector informal incluyen: técnicas de producción simples, nivel de cualificación (formal) poco elevado, pequeña escala de actividad, débil capacidad de acumulación, propiedad familiar, relaciones sociales sin salario, operaciones al margen de la ley, débil protección del trabajo, entre otros.

En América Latina y el Caribe, según la OIT (2016) hay al menos 130 millones de personas en la región que trabajan en condiciones de informalidad. La informalidad en grupos de bajos ingresos llega incluso a sobrepasar el 70\% de la población. En el Ecuador, según datos del Instituto Nacional de Estadísticas y Censos (2015), aproximadamente el 39\% de la población económicamente activa pertenecería al sector informal (según la última actualización al método) o 53\% de la población económicamente activa (según el método anterior).

Esta diferencia se debe a que la metodología para recopilar estadísticas del sector informal varió en los últimos años ${ }^{9}$ en cuanto a: tipos de encuestas de empleo, desempleo y subempleo, incremento de tamaño de la muestra, nuevos marcos conceptuales de clasificación, entre otros. Con respecto al último punto, el sector informal se redefinió en Ecuador a partir de los criterios de: 1) posesión de registro ante la autoridad tributaria (denominado Registro Único de Contribuyentes - RUC) y 2) de la obli-

${ }^{9}$ Ver: INEC (2015): Actualización metodológica: Empleo en el sector informal y la clasificación de los ocupados según sectores 
gación de llevar registros contables completos, según el concepto de "empresas del sector informal" que estableció la Organización Internacional del Trabajo (s.f.) en su publicación titulada "Medición de la Economía Informal".

La actualización más reciente de la metodología utilizada en Ecuador (julio, 2015) señala que, en concordancia con la legislación nacional vigente aun cuando las personas, hogares o empresas no lleven registros contables completos, la sola tenencia del RUC les caracterizaría como parte del sector formal. La justificación de la actualización de la metodología se da al asumir que las personas, hogares o empresas que posean RUC harían declaraciones tributarias, emitirían comprobantes de venta y pagarían impuestos, con lo cual ya no serían considerados "informales". El Instituto Ecuatoriano de Estadísticas y Censos señala, por tanto, que la metodología anterior (empleada hasta marzo de 2015) sobreestimaba la informalidad. Sin embargo, también podría ocurrir lo contrario, es decir que lo que se estaría sobreestimando sería la formalidad pues a través de este método y se dejaría de lado las otras racionalidades presentes en la economía popular y solidaria (vínculos sociales y familiares, lógicas de reciprocidad o bien economías mestizas como se analizará en el último apartado), por tanto, se caería en el error de disasociar este tipo de economía del contexto en el que se presenta.

Las lecciones aprendidas al momento de procurar replicar los modelos de economía social de países del norte en el sur demuestran, en muchos casos, una alta ineficacia de los proyectos por parte de los ejecutores (Estado, ONGs, etc). Esta situación se debe al escaso conocimiento de las necesidades de la población en cuestión, así como de las redes que regulan la vida social. Estas redes de reciprocidad, según Fonteneau (en Defourny J. et. al. (2006) tiene una función importante que es ser un contrapeso al carácter intrínsecamente desigual de la organización social. Son entonces, formas específicas de redistribución que reflejan las dinámicas conflictivas de construcción de estos modelos comunitarios. Dicho esto, tener en Ecuador el concepto de economía popular y solidaria y no obstante esto, aplicar políticas públicas que se limitan a utilizar en la recolección de estadísticas el concepto de sector informal resulta contradictorio. 


\section{PERCEPCIONES Y NARRATIVAS CONTRADICTORIAS DEL ACUERDO COMERCIAL MULTIPARTES ENTRE COLOMBIA, PERÚ, ECUADOR Y LA UNIÓN EUROPEA}

Estudiar el comercio justo, así como la economía social y la economía populary solidaria en el marco del Acuerdo de Comercio Multipartes Ecuador-UE nos servirá para seguir ejemplificando, primero, la polisemia de los términos y luego, las distintas narrativas que se construyen en torno a la significación y simbolización de este Acuerdo en los distintos territorios.

\subsection{Antecedentes y justificación de la firma del acuerdo}

Los acercamientos entre la Unión Europea y la Comunidad Andina (Colombia, Perú, Ecuador y Bolivia) empezaron en 2007. No obstante, Bolivia dejó las negociaciones ese mismo año al alegar que un acuerdo comercial iría en contra de las regulaciones andinas de propiedad intelectual, entre otros temas. Un año después Ecuador hizo lo mismo. Los representantes de la Unión Europea dejaron abierta la posibilidad que se vuelvan a abrir las negociaciones a futuro con Ecuador y Bolivia.

Para el Ecuador, el proceso de restablecimiento de las negociaciones no fue sencillo dado que el Presidente Rafael Correa insistía, en ese entonces, que el acuerdo alcanzado entre la UE y Colombia y Perú no podía ser igual al de Ecuador. En lugar de un tratado de libre comercio, el líder ecuatoriano afirmó que las dos partes necesitaban asegurar un acuerdo de "mutuo beneficio" (International Center for Trade and Sustainable Development, 2014).

El Ecuador, al igual que otros países de la Comunidad Andina, se beneficia de preferencias arancelarias en el mercado europeo bajo el Sistema Generalizado de Preferencia (SGP), específicamente bajo un incentivo que se direcciona a promocionar el buen gobierno y el desarrollo sostenible denominado SGP+. El Ecuador iba a perder estas preferencias en diciembre de 2014 después de ser categorizado como un país de "ingresos medios altos" por tres años consecutivos según el método de clasificación Atlas del Banco Mundial, lo cual significaba que bajo la normativa europea Ecuador ya no se categoriza como un país apto para recibir estos beneficios comerciales.

La Unión Europea constituye el principal mercado para las exportaciones no petroleras de Ecuador y el tercer mercado más importante para exportaciones de manufacturas. En este contex- 
to, el Presidente Correa ${ }^{10}$ manifestó en 2014 que los costos de no contar con este Acuerdo habrían sido muy altos para el país, al considerar que los países vecinos y muchos de nuestros principales competidores comerciales ya firmaron acuerdos comerciales con la UE. En la misma línea, el Ministerio de Comercio Exterior (2014) en una publicación explicativa sobre el Acuerdo Comercial con la Unión Europea señala que "la ausencia de un acuerdo comercial afectaría directamente a la producción nacional, las exportaciones, el empleo directo e indirecto y la generación de divisas. Los impactos se extenderían a varios sectores sociales y a grandes y medianas empresas, así como a los actores de economía populary solidaria".

Como parte de los acuerdos que se alcanzaron durante las rondas de negociación, se aceptó que se renueve el $\mathrm{SGP}+$ para Ecuador hasta que el nuevo acuerdo comercial entre en vigor. En ausencia de las preferencias arancelarias se necesitaba de una subvención de aproximadamente 332 millones de dólares a fin de que Ecuador sea competitivo en este mercado o, según estudio de la CEPAL citado en el documento que se menciona, la no firma del Acuerdo con la

${ }^{10}$ Telesur (2014) afirma explícitamente en el titular de su artículo "Document Reveals EU Bullied Ecuador IntoTradeAgreement" que Ecuador ha sido forzado a adherirse al Acuerdo Comercial.
UE ocasionaría una disminución del 5,3\% de las exportaciones ecuatorianas hacia este destino en 2020 (Ministerio de Comercio Exterior, 2014).

El Acuerdo Comercial Multipartes Ecuador-Unión Europea que se prevé será ratificado en la segunda mitad de 2016.

\subsection{Múltiples narrativas sobre lo que significa el acuerdo comercial negociado entre Ecuador y la UE}

Lo que se analizó hasta el momento, la polisemia del término comercio justo, así como de la economía populary solidaria y la economía social. También se toma en consideración que la UE, a pesar de establecer que el comercio justo forma parte de su política de cooperación internacional al mismo tiempo lo relega al ámbito del tercer sector o bien de compromisos de tipo voluntario por los que organizaciones de la sociedad civil europea pueden o no optar. Asimismo, se observa que tanto el comercio justo como la economía popular y solidaria forman parte de un proyecto político para el Estado ecuatoriano, con consecuencias tanto internas como externas y con múltiples dificultades al momento de poner en práctica retóricas posneoliberales en un mundo globalizado e interdependiente. De aquí surgen y se pueden explicar, las contradicciones al momento de definir el significado del Acuerdo Comercial entre Ecuador y la UE. 


\section{ACUERDO DE COMERCIO PARA EL DESARROLLO}

Después de casi cuatro años de trabajo, se cerró en 2014 un acuerdo entre Ecuador y la Unión Europea que, según Francisco Rivadeneira, entonces Ministro de Comercio Exterior, maximiza las oportunidades, reduce los costos y respeta los mandatos constitucionales. De hecho, Rivadeneira afirmó que se logró un Acuerdo muy cercano a lo que el país denominó como un Acuerdo Comercial para el Desarrollo, cuyos elementos principales constituyen: el mantenimiento de la soberanía nacional en el manejo de sus políticas públicas y la preservación de fuentes de empleo en los sectores productivos, con especial énfasis en las micro, pequeñas y medianas empresas, así como de los actores de la economía popular y solidaria ${ }^{11}$; entre otros (Ministerio de Comercio Exterior, 2014).

En la misma línea, el ex-Ministro de Comercio Exterior (2014) enfatizó que el Acuerdo Comercial con la Unión Europea, a pesar de ser el mismo acuerdo que negociaron Colombia y Perú, tiene especificidades para el Ecuador que hacen posible que se mantenga y respete

\footnotetext{
${ }^{11}$ Con respecto a la contratación pública, el Ministerio de Comercio Exterior de Ecuador (2014) señala que todos los programas y proyectos de contratación pública a favor de las PYMES y de las Asociaciones de Economía Popular y Solidaria fueron excluidos de las coberturas del Acuerdo comercial.
}

el modelo de desarrollo nacional y por tanto le confiere "plena originalidad".

Rafael Correa en una entrevista en Telesur publicada por la SECOM y la Presidencia de la República en 2014, señaló que "en el Ecuador no creemos en el libre comercio (...) pero sí creemos en el comercio para mutuo beneficio". También hizo hincapié en las presiones que tenía Ecuador frente a la caducidad del SGP. Señaló "Se negoció muy duramente, desde el principio pusimos líneas rojas que no íbamos a traspasar, proteger el sistema de compras públicas, proteger los pequeños productores agrícolas, proteger la pequeña y mediana industria".

Se justifica, sobre todo a través de los Art. 13 y 324, la diferencia fundamental entre un tratado de libre comercio y un acuerdo de comercio para el desarrollo. Así, en el Art. 13 del Acuerdo referente a las funciones del Comité de Comercio, Ecuador hizo una precisión respecto a lo que se negoció entre la UE con Perú y Colombia y añadió que además que dicho comité examine el impacto del Acuerdo sobre las micro, pequeñas y medianas empresas también se incorpore a las Asociaciones de Economía Popular y Solidaria (Ministerio de Comercio Exterior, 2014).

Asimismo, desde la perspectiva de Villagómez, M. (s.f.), Embajador de Carrera del Servicio Exterior Ecuatoriano en su artículo titulado "Acuerdo Comer- 
cial Multipartes Ecuador-Unión Europea", el Art. 324 del Acuerdo referente a la Asistencia Técnica y al Fortalecimiento de la Capacidad Empresarial marcaría la diferencia del Acuerdo Multipartes Ecuador- UE de un Tratado de Libre Comercio dado que señala todas las ventajas en el ámbito de la cooperación para el comercio derivadas de "este importante instrumento internacional".

Cabe mencionar que el Art. 324 no constituye una reforma al Acuerdo firmado entre Colombia, Perú y la Unión Europea. Este apartado ya constaba en las negociaciones anteriores concluidas en 2011 y aprobadas por los Ministros de Comercio de la UE el año siguiente para que entre en vigencia a mediados de 2013 en Colombia y Perú. Este apartado busca fomentar la cooperación a fin de que se expandan las oportunidades y se obtengan mayores beneficios para las partes, en el marco del enfoque de desarrollo económico sostenible que permita alcanzar mayores niveles de cohesión social y reducir la pobreza. Así, dentro de las iniciativas de cooperación relacionadas con el comercio justo se puede señalar:

1) Promover el desarrollo de las MIPYMES, al usar al comercio como una de las herramientas para la reducción de la pobreza

2) Promover al comercio justo $y$ equitativo, al facilitar el acceso a los bene- ficios del Acuerdo por todos los sectores productivos, en particular los más débiles

\section{TRATADO DE LIBRE COMERCIO}

Una vez concluidas las negociaciones con Ecuador, la Comisión Europea afirmó que sí se hicieron cambios limitados al acuerdo existente con Colombia y Perú. Sin embargo, estos cambios no representan mayores diferencias en el alcance del acuerdo que ya se estableció previamente (International Center for Trade and Sustainable Development, 2014).

El mismo Acuerdo viene a ser denominado tratado de libre comercio por Colombia (Delegación de la Unión Europea en Colombia, 2012), donde en el portal web oficial se indican las ventajas del acceso a mercados que se derivan de este Acuerdo como el objetivo principal de la negociación. Asimismo, el Ministerio de Comercio Exterior y Turismo de Perú (2011) al igual que la UE (2013) afirman que negociaron y concluyeron un tratado de libre comercio que mejoró el acceso a mercados de productos agrícolas peruanos en el mercado europeo. En el portal de la Unión Europea (2013) dedicado a la región andina, se promociona la decisión del Consejo que permitió la conclusión y firma del protocolo que permitió a Ecuador adherirse al tratado de libre comercio que se negoció entre la Unión Europea y Colombia y Perú. 
En una entrevista a Katiuska King realizada en 2012 por Tamayo E., publicada en América Latina en Movimiento, la exministra coordinadora de la Política Económica de Ecuador afirma que, el Acuerdo de Comercio Multipartes con la UE es un sinónimo de un tratado de libre comercio y por tanto iría en contra del régimen del Buen Vivir que establece la
Constitución. Señala King, "Un tratado de libre comercio es la expresión máxima de la desregulación de mercados o de las políticas neoliberales que se aplican, por lo tanto, habría una inconsistencia si se llega a firmar, porque esto no iría en beneficio del país y no permitiría plantear una estrategia de desarrollo distinta."

\section{4. ¿CÓMO DIBUJAR FRONTERAS CONCEPTUALES ENTRE LA ECONOMIIA DE LIBRE MERCADO Y LA ECONOMÍA POPULAR Y SOLIDARIA?}

El Acuerdo de Comercio Multipartes Ecuador-Unión Europea sirve como caso para reflexionar sobre la polisemia de las economías alternativas en el marco de proyectos políticos posneoliberales, dentro de lo que se denomina como la nueva izquierda latinoamericana en su afán por diferenciarse de proyectos políticos neoliberales. La utilización del concepto de economía popular y solidaria, así como del comercio justo en el marco de las negociaciones realizadas con Europa hace posible identificar cómo se construyen múltiples narrativas en torno a un documento (el Acuerdo) que desde sus inicios se concibió como un tratado de libre comercio en concordancia con la política comercial europea.

En este contexto y a manera de conclusión, conviene preguntarse cómo dar coherencia al proyecto político de Ecuador para que las nociones de economía popular y solidaria y de comercio justo, constituyan realmente elementos diferenciadores dentro de la política interna y externa. Se pretende a través de esta reflexión contribuir al debate académico a través de fundamentos microteóricos de la Economía Política Internacional al visibilizar las implicaciones de la globalización económica, no únicamente enfocada en el Estado y el mercado, sino en las complejas redes sociales (institucionalizadas o no) que están presenten en los territorios.

El principal problema de estudiar el llamado sector informal desde enfoques tradicionales del desarrollo (teoría neoclásica y la corriente estructuralista), implica asumir que muchas de las ca- 
racterísticas anteriormente mencionadas son el resultado únicamente de la "ausencia de trabajo formal" $y$, por ende, de "sectores modernos" dentro y la economía. Así, Lewis (1954), por ejemplo, supone que el desarrollo económico se da gracias a la acumulación de capital en el sector formal (léase "urbano" o "industrial") mientras que el sector tradicional (es decir "rural" y "agrícola"), sirve para asegurar la existencia de oferta de mano de obra. (Kuhnen, 1987).

Desde esta perspectiva alternativa, la unidad económica de base no sería la empresa sino las redes sociales en su conjunto. Se trataría en este caso, del estudio no únicamente de la economía de libre mercado, sino también de la "acumulación relacional" regida por el principio de la reciprocidad. (Fonteneau en Defourny et. al. 2006). Esto es lo que Verhelst (1996) (en Defourny et. al. 2006) denomina economías mestizas como un conjunto de lógicas combinadas tanto capitalistas como no capitalistas, caracterizadas por una superposición de valores (una mezcla de lógicas mercantiles con lógicas de reciprocidad, o bien de lógicas formales e informales). Finalmente, otra característica de la economía popular estaría dada por la diferencia fundamental en el tema de la productividad. Chayanov distingue que mientras las empresas buscan aumentar la cantidad de producto por trabajador, las economías de trabajo pretenden aumentar el ingreso neto por trabajador. (Arias y Racines, 2015). La lógica de las empresas de economía popular antes que situarse en formas de acumulación de tipo capitalista, se desarrolla para dar respuesta a las necesidades de sus miembros. Esto da una particular lógica de funcionamiento a este tipo de unidades, que puede ser estudiada a través de variables como: el tipo de acumulación, la redistribución de los excedentes y las relaciones de trabajo.

De esta manera, los supuestos que: 1) la mano de obra excedentaria sea absorbida poco a poco por el "sector moderno". (Van Dijck, 1986 en Defourny et. al. 2006); 2) que el sector informal no es más que un espacio temporal de "espera" (Rouband, 1994 en Defourny et. al. 2006) de otros tipos de trabajos o 3) que el sector informal actúa como un regulador de la crisis que caracteriza a los Ilamados países en desarrollo, no se cumplirían necesariamente, o no, por lo menos en todos los casos. El mayor riesgo de naturalizar estos supuestos es dejar de lado la posibilidad de comprender que la economía informal va más allá del anhelo de "formalizarse" y representa formas diferentes de organización social.

De todo lo anterior se infiere, por tanto, que de manera discursiva el concepto de economía popular y solidaria en Ecuador procura establecer fronteras que lo diferencian del concepto de 
economía de libre mercado. Sugiero en este artículo, que la economía popular y solidaria se estudie tomando en consideración los planteamientos de Gudeman (1986) (en Walsch, 2013) en lo que respecta a comprender que la economía es un sistema cultural y de Vega (2001) (en Walsch, 2013) desde la perspectiva de las teorías poscoloniales a fin de poder comprender y posteriormente deconstruir las narrativas sobre la modernización y el progreso, por ejemplo, así como develar contenidos totalizadores que se imponen en contextos históricos y realidades no-europeas. Al seguir esta lógica, la economía debería estudiarse a partir de los contextos y particularidades del territorio en las que se manifiesta, pues, como afirma Quijano (2013) (en Walsch, 2013) las categorías occidentales dificultan muchas veces el entendimiento de la economía en sus múltiples manifestaciones e incluso producen distorsiones interpretativas".

\section{CONCLUSIONES}

Las tres críticas que se plantearon al inicio de este artículo sirven para explicar las dificultades en la aplicación de estrategias para diferenciar la economía populary solidaria de la economía de libre mercado dentro del proyecto político posneoliberal en Ecuador. Esto se hace evidente frente a una política comercial ecuatoriana que en el transcurso de los últimos años da un giro, al pasar de un aparente aislacionismo a un involucramiento activo en el comercio internacional, a través de la firma de un tratado de libre comercio con un socio comercial de gran relevancia para el país. Esto levanta cuestionamientos acerca de si la nueva izquierda latinoamericana plantea o no propuestas diversas, tanto frente a las agendas neoliberales como a las de la vieja izquierda del siglo XX. Vuelven a surgir en este contexto, preocupaciones sobre la pluralidad de la izquierda mundial y sobre el movimiento por una globalización alternativa que en el caso latinoamericano tomaron fuerza a finales de la década de 1990 y en la primera década del siglo XXI.

Así, con respecto a la primera crítica se señala que la polisemia de los términos comercio justo, economía popular y solidaria y economía social, no únicamente en Ecuador sino también en la Unión Europea, se debe a que no existe una definición unívoca de los conceptos. Resulta en extremo complejo dibujar fronteras conceptuales entre el libre comercio y el comercio justo si el último concepto varía de significado. En este 


\section{LA ECONOMÍA POPULAR Y SOLIDARIA COMO PROYECTO POLÍTICO \\ POSTNEOLIBERAL DE ECUADOR. CASO: ACUERDO COMERCIAL UNIÓN

sentido, se destaca cómo el comercio justo, desde la perspectiva europea puede responder, en primer lugar, a los ideales de la economía social fomentada históricamente por el tercer sector y luego, a la política comercial europea que lo considera desde la perspectiva de iniciativas de tipo voluntario que puede acoger la sociedad civil o bien desde el enfoque de las barreras para-arancelarias al comercio internacional (también de tipo discrecional).

En la misma línea se señala las notorias diferencias entre el concepto de economía social y el de economía popular y solidaria pues este último, se constituyó en la primera década del nuevo milenio en un proyecto político en Ecuador dentro de la denominada nueva izquierda latinoamericana que, buscaba diferenciarse de proyectos políticos anteriores. Los matices que abarca la economía popular y solidaria como proyecto político son muchos pues incluyen desde nociones diferenciadoras como la cooperación y la reciprocidad, presentes de manera discursiva en políticas internas y externas, hasta elementos que se toman de la economía neoclásica como la noción del sector informal (igualmente ambigua a pesar de las definiciones dadas por la Organización Internacional del Trabajo así como por las definiciones cambiantes que el Estado ecuatoriano da al término).
La segunda crítica se plantea en referencia a las distintas narrativas que, como resultado de la ambigüedad conceptual, se suscitan en el marco del Acuerdo de Comercio Multipartes entre Ecuador y la UE. Se observa que el Acuerdo desde la perspectiva de instituciones gubernamentales ecuatorianas se asemeja a un Acuerdo de Comercio para el Desarrollo, (instrumento originalmente conceptualizado por la SENPLADES como alternativa a los tratados de libre comercio), que procura ante todo salvaguardar la soberanía y autonomía del Ecuador y su modelo de desarrollo. La retórica utilizada por los distintos representantes del gobierno apunta a que el Acuerdo negociado con la UE se distinga conceptualmente de un tratado de libre comercio. Se pretende, así, guardar una cierta coherencia con el proyecto político post-neoliberal y también con los mandatos constitucionales. Por otro lado, el mismo Acuerdo leído desde el resto de actores involucrados: Colombia, Perú y la UE es denominado tratado de libre comercio ya que no contradiría las políticas comerciales vigentes en dichos países.

Finalmente, como respuesta a las críticas anteriormente planteadas, el último apartado recoge elementos conceptuales que pretenden aclarar, en la medida de lo posible, las fronteras que permitirían diferenciar a la economía po- 
pular y solidaria de la economía de libre mercado. Se identifica en este sentido, las dificultades metodológicas de estudiar a las relaciones económicas en función de teorías clásicas y neoclásicas basadas únicamente en la Teoría de la Elección Racional y deja de lado lógicas distintas que permitan comprender y dibujar líneas divisorias entre la economía de libre mercado y las economías alternativas. Todavía quedan, sin embargo, muchas más fronteras por definir tanto en el ámbito jurídico como en el de aplicación de políticas públicas, así como en el rol del tercer sector, en lo que respecta la pluralidad del sentido de la economía.

El riesgo de no definir las fronteras conceptuales entre economía de libre mercado y economía popular y solidaria, en el marco de acuerdos de comercio multipartes - como el Acuerdo de Comercio entre la UE y el Ecuador - podría significar para los actores de la economía populary solidaria una pérdida importante. Así, por ejemplo, concebir al comercio justo únicamente desde la perspectiva del etiquetado y del precio justo, sin tomar en cuenta la compleja red de motivaciones, valores y prácticas sociales que conllevan las economías alternativas tendría múltiples implicaciones.

Por tanto, como afirman Gibson-Graham (2004) es importante recordar la importancia de "afirmar una lógica de la diferencia y de la posibilidad" para contraponer las tendencias homogeneizadoras globales al igual que las generalizaciones teleológicas de la economía política; solo así se podrán visibilizar las actividades ocultas y las alternativas que están presentes por todos lados. De esta forma, las mismas autoras plantean la importancia de entender a las economías locales como lugares con identidades y capacidades económicas altamente específicas y no como simples nódulos de un sistema capitalista mundial.

Dada la arquitectura del comercio internacional vigente, los acuerdos comerciales bilaterales, así como los acuerdos comerciales multipartes y sobre todo los nuevos mega-acuerdos comerciales, representan un punto de referencia al momento de entender cómo se reinventaría (utilizando las palabras de Defourny, Develtere y Fonteneau) la economía alternativa en los distintos países, frente a estas nuevas mutaciones del capitalismo.

Al tomar en consideración, primero el contexto de la denominada crisis del Estado de Bienestar, las altas tasas de desempleo, las deudas soberanas, así como la exclusión social (evidenciada sobre todo en lo que se denomina hoy el "cuarto mundo") y luego, el subempleo y empleo informal, la desigualdad, la inseguridad alimentaria, la limitada satisfacción de necesidades básicas (salud, educación, ambiente), entre otros, 
somos testigos y cocreadores de la resignificación de la economía social y de la economía populary solidaria.

Una vez que se analiza esto, es importante no olvidar que "las personas participan simultáneamente en más de una economía y a menudo en más de una comunidad cultural" (Bird-David, 2004 en Defourny et. al. 2006). Por tanto, difícilmente se podría afirmar que en la actualidad los agentes de la economía popular y solidaria en Ecuador basan sus actividades en función de los principios de la reciprocidad, la solidaridad y la cooperación exclusivamente. Esto complejiza aún más el estudio y la delimitación de fronteras entre las lógicas de la economía de libre mercado y las lógicas de las economías alternativas.

Quedan otros temas abiertos también como la burocracia y la experticia en el marco de la transformación estatal al igual que el pasado y el futuro de la gobernanza liberal y posneoliberal en Ecuador, así como las posibilidades de imaginarios políticos y económicos alternativos. Finalmente, la utilización del concepto de izquierda como categoría analítica queda en entredicho dada la continuidad y profundización de políticas de corte neoliberal en América Latina o del regreso de la derecha.

\section{BIBLIOGRAFÍA}

Arias, S. y Racines, F (2015): Cadenas Sociales de Valor para la Integración Suramericana. Seminario sobre Proyectos de Infraestructura y Cadenas Sociales de Valor. UNASUR Quito. IIRSA. Recuperado de: http://www.iirsa .org/admin _iirsa_web/Uploads/Documents/ unasur_quito15_acosta_discurso.pdf

Asamblea Constituyente de Bolivia (2008): Constitución Bolivia. Recuperado de: http:// www.comunicacion.gob.bo/sites/default/files/ docs/Nueva_Constitucion_Politica_del_Estado_Boliviano_0.pdf Asamblea Nacional del Ecuador (2008): Constitución de la República del Ecuador. Recuperado de: http:// www.asambleanacional.gob.ec/ sites/default/files/ documents/ old/constitucion_de_bolsillo.pdf Bateson, G. (1972). Steps to an Ecology of Mind: Collected Essays in Anthropology, Psychiatry, Evolution, and Epistemology. University Of Chicago Press.

Browning, C. (2015): "Nation Branding, National Self-Esteem, and the Constitution of Subjectivity in Late Modernity". Foreign Policy Analysis. Volume 11. Issue 2. P 195-214

Calvo, C. (2009): La "Nueva" Izquierda Latinoamericana. Revista Reflexiones 
88 (1): 55-65, ISSN: 1021-1209. Recuperado de: http://www.redalyc.org/pdf/729/ 72912559004. pdf

Chávez, D., Rodríguez, C. y Barret, P. (2008): La nueva izquierda en América Latina. Recuperado de: http://latinoamerica.sociales.uba. ar/files/2015/01/Chavez-Garavito-y-Barrett-La-nueva-izquierda-en-Am\%C3\%A9rica-Latina.pdf Comisión Europea (1999): Comunicación de la Comisión al Consejo, de 29 de noviembre de 1999, relativa al comercio justo. Recuperado de: http://eur-lex.europa.eu/legal-content/ES/TXT/?uri=URISERV\%3Ar12508

Comisión Europea (2014): Comprender las políticas de la Unión Europea. Comercio. Recuperado de: http:// europa.eu/pol/pdf/flipbook/es/ trade_es.pdf

Comisión Europea (2014): EU and Ecuador publish text of trade agreement. Recuperado de: http:// trade.ec.europa.eu/doclib/press/ index.cfm?id=1156

Comisión Europea (2009): FairTrade and non-governmentaltrade-relatedsustainability assurance schemes. Recuperado de: http://eur-lex. europa.eu/legal-content/EN /TXT /?uri=URISERV:dv0004

Coraggio, J. (2011): Economía Social y Solidaria. El trabajo antes que el capital. Editorial AbyaYala. Quito - Ecuador. Recuperado de: http:// www.coraggio economia.org/jlc/ archivos\%20para\%20descargar/ economiasocial.pdf

Delegación Unión Europea en Colombia (2012): Acuerdo Comercial Colombia-Unión Europea. Recuperado de: http://www.tlc.gov.co/publicaciones. php? $\mathrm{id}=18028$

Didier, K. (2010): TerritoiresProduits par l'industrie miniere et rupture de filiation ouvriere. Université Catholique de Louvain. Presses Universitaires de Louvain. Belgique. Recuperado de: http://www.congoforum.be/ upldocs/THESE\% 20DE\%20KILONDO\%20Novembre\%202010. pdf

Dieterich, H.(2007): Hugo Chávez y el Socialismo del Siglo XXI, Segunda Edición Revisada y Ampliada. Edición Digital. Recuperado de: http://www.rebelion .org/ docs/121968.pdf

Defourny J et al. (2006): L'économie sociale au Nord et au Sud. Deuxième Tirage. Éditions De Boeck Université. Bruxelles.

Fair Trade Advocacy Office (2016): Fair trade and the EU. Recuperado de: http://www. fairtrade-advocacy. org/fair-trade-and-the-eu

Gibson-Graham, J.K. (2004): Surplus possibilities. Postdevelopment and community economies. Recupe- 
rado de: http://law.uvic.ca/demcon/victoria_colloquium /documents/Surplus_Possibilities.pdf Instituto Nacional de Estadísticas y Censos (2015): Actualización metodológica: Empleo en el sector informal y clasificación de los ocupados según sectores. Recuperado de: http://www.ecuadorencifras.gob.ec/documentos/ web-inec/EMPLEO/2015/Junio-2015/Metogologia_Informalidad/notatecnica.pdf

International Center for Trade and Sustainable Development (2014): EU, Ecuador Finalise Trade Pact. Recuperado de: http://www.ictsd.org/ bridges-news/bridges /news/ eu-ecuador-finalise-trade-pact

Kuhnen, F. (1987): Causes of underdevelopment and concepts for development. The Journal of Institute of Development. Recuperado de: http://www2.fiu.edu/ ereserve 1010029280-1.pdf

Ministerio de Comercio Exterior del Ecuador (2014): Acuerdo Comercial Ecuador - Unión Europea. Recuperado de: http://www.comercioexterior.gob.ec/wp-content/ uploads/downloads/2015/04/ CARTILLA-UNION-EUROPEA- 1 . pdf

Ministerio de Comercio Exterior del Ecuador (2014): Noticias "Se cerró el Acuerdo entre Ecuadory la Unión Europea". Recuperado de: http:// www.comercio exterior.gob.ec/ se-cerro-el-acuerdo-entre-ecuador-y-la-ue/

Ministerio de Comercio Exterior del Ecuador (2014): Paquete de cierre de la negociación comercial Ecuador-Unión Europea. Recuperado de: http://www. comercioexterior.gob.ec/wp-content/ uploads/2014/09/TEXTOS-NEGOCIADOS-EN-EL-ACM.pdf

Ministerio de Comercio Exterior y Turismo de Perú (2011): Acuerdo Comercial entre entre Perú y la Unión Europea. Recuperado de: http:// www.acuerdos comerciales.gob. pe/index.php?option=com_ content\&view=category\&layout=blog\&id $=50 \&$ ltemid $=73$

Nelms, T. (2015): Making popular and solidarity economies in dollarized Ecuador. Money, Law and the social after neoliberalism. University of California Irvine.

Organización Internacional del Trabajo (2016): Economía Formal en América Latina y el Caribe. Recuperado de: http://www.lo.org/americas/ temas/econom\%C3\% ADa-informal/lang--es/index.htm

Organización Internacional del Trabajo (2014): Evolución del empleo informal en Ecuador 2009-2012. Perú, 
Lima: OIT - FORLAC (Programa de Promoción de la Formalización en América Latina y el Caribe). Recuperado de: http://www.lo .org/wcmsp5/groups/public/--americas/---ro-lima/documents/ publication/wcms_245616.pdf

Organización Internacional del Trabajo (s.f): Medición de la Economía Informal. Recuperado de: http:// www.ilo.org/wcmsp5/groups/ public/---ed_emp/---emp_policy/documents/publication/ wcms_229450.pdf

Palmer, R. (2004): The informal economy in Sub-Saharan Africa: unresolved issues of concept, character and measurement. Centre for African Studies. Edingburgh University. Recuperado de: http://www.cas. ed.ac.uk/_data/assets/pdf_file /0020/27362/No_098_the_informal_economy_in_sub-saharan_ africa-_unresolvec.pdf

Parlamento Europeo (2006): Resolución sobre "Comercio Justo y Desarrollo". Recuperado de: http://www.europarl.europa.eu/sides/getDoc. do?pubRef=-//EP//NONSGML+TA+P6-TA-2006-0320+0+DOC+P$\mathrm{DF}+\mathrm{V} 0 / / \mathrm{ES}$

Parlamento Europeo (2012): Trade in Public Procurement in the EU. Library of the European Parliament. Re- cuperado de:http://www.europarl.europa.eu/RegData /bibliotheque/briefing/2012/120334/ LDM_BRI(2012)120334_REV1_ EN.pdf

Presidencia de la República (2014): Presidente Correa: Se ha logrado con la Unión Europea un acuerdo bastante conveniente para Ecuador. Recuperado de: https ://www. youtube.com/watch?v=GhruGQsYmEM

Secretaría Nacional de Planificación y Desarrollo (2009): Plan Nacional del Buen Vivir (2009-2013). Recuperado de: http://www.planificacion.gob.ec/plan-nacional-parael-buen-vivir-2009-2013/

Secretaría Nacional de Planificación y Desarrollo (2013): Plan Nacional del Buen Vivir (2013-2017). Recuperado de: http://documentos. senplades.gob.ec/Plan\%20 Nacional\%20Buen\%20Vivir\%20 2013-2017.pdf

Secretaría Técnica de Cooperación Internacional (2015): Diccionario de Cooperación Internacional. Recuperado de: http://www. cooperacioninternacional. gob.ec/wp-content/uploads/ downloads/2015/05/Diccionario-de-Cooperacio\%CC\%81n-Internacional.pdf 
Secretaría Técnica de Cooperación Internacional (2012): Revista Cooperamos N4 "Cambio Climático y Justicia Ecológica. Quinta Edición. Recuperado de: http://www.cooperacioninternacional.gob.ec/ wp-content/uploads/downloads /2014/04/Revista_Cooperamos_ edicion-5.pdf

Superintendencia de Economía Popular y Solidaria (2012): Reglamento a la Ley Orgánica de Economía Popular y Solidaria. Recuperado de: http://www. seps.gob.ec/documents/20181/25522/Reglamento\%20a\%20la\%20Ley\%20Organica\%20del\%20sistma\%20de\%20 economia\%20popular\%20y\%20 solidaria\%20DE1278-23ago2012. pdf/a86ec15b-a269-4ab6-8772083ef6b80dfe

Tamayo, E. (2012): Acuerdo de Comercio con la UE. ¿Ecuador entre la espada y la pared?. América Latina en Movimiento. Recuperado de: http://www.alainet .org/es/active/53174\#sthash.klCEqhSh.dpuf

Telesur (2014): Document Reveals EU Bullied Ecuador IntoTradeAgreement. Recuperado de: http:// www.telesurtv.net/english/ news/Document-Reveals-EU-BuIlied-Ecuador-Into-Trade-Agreement-20141009-0050.html
Unión Europea (2012): Acuerdo Comercial Multipartes Unión Europea-Colombia y Perú. Recuperado de: http:// eur-lex.europa.eu/legal-content/ EN/TXT/?uri =OJ:L:2012:354:TOC

Unión Europea (2014): Comprender las políticas de la Unión Europea: Cooperación Internacional y Desarro/lo. Recuperado de: http://europa. eu/pol/pdf/flipbook/ es/ development_cooperation_es.pdf

Unión Europea (2013). Trade. Andean Community. Recuperado de: http://ec.europa.eu/trade/ policy/countries-and-regions/regions/andean-community/

Villagómez, Mentor (s.f): Acuerdo Comercial Multipartes Ecuador Unión Europea. Recuperado de: https:// eulacfoundation.org/es/system/ files/Acuerdo\%20 comercial\%20 Ecuador-UE.pdf

Walsh, C. (2013): Pedagogías decoloniales. Prácticas insurgentes de resistir, re (existir) y (re)vivir. Tomo I. AbyaYala. Quito. 